\title{
Driver Fatigue Detection Method Based on Eye Multi-Feature Fusion
}

\author{
Qinghua Liu ${ }^{1, *}$, Haixiao Zhong ${ }^{2}$, Xuehan Zhao ${ }^{2}$ and Lu Sun ${ }^{1}$ \\ ${ }^{1}$ School of Engineering and Applied Science, Princeton University, Princeton, America \\ ${ }^{2}$ School of Computer Science, Jiangsu University of Science and Technology, Zhenjiang, China \\ ${ }^{*}$ Corresponding author
}

\begin{abstract}
In order to improve the accuracy of driver fatigue detection, this paper proposes a new fatigue detection method based on random forest regression model and integrate eye fatigue surveillance method for multiple characteristic parameters. First, we use the features of simple class Haar to cascade algorithm Adaboost, aiming at carrying out quick face location detection. To solve the problem of low accuracy and high misclassification rate when uses the conventional method PERCLOS (percentage of eyelid closure over the pupil over time) via single parameter judgment, the paper increases four new parameters while extracting the eye fatigue characteristics and uses the classification and regression tree(CART) to judge whether the driver is awake or tired. The experimental results show that the accuracy rate of the driver's fatigue test is $96.7 \%$, the error rate of the driver is $1.7 \%$ when the driver is in the fatigue state, the processing speed of our driver fatigue detection system is about $\mathbf{3 0}$ frames per second, it can make a judgment about the driver's fatigue state in about 10 seconds, which owns higher accuracy and real-time.
\end{abstract}

Keywords-face detection; random forest regression model; PERCLOS; CART; fatigue detection

Effective prevention of fatigue driving is of great significance to reduce traffic accidents and it is an important measure to prevent fatigue driving by real-time detection and warning of driver's fatigue state.In this paper, based on the previous research, combined with the research result in the field of facial feature point registration, a new driver fatigue detection is proposed. The general idea is shown in Figure 1. Firstly, the image of the driver face is collected and preprocessed and the face is detected with the Viola-Jones face detection algorithm[1]. Then the feature points are located and finally the fatigue characteristic is extracted and judged.

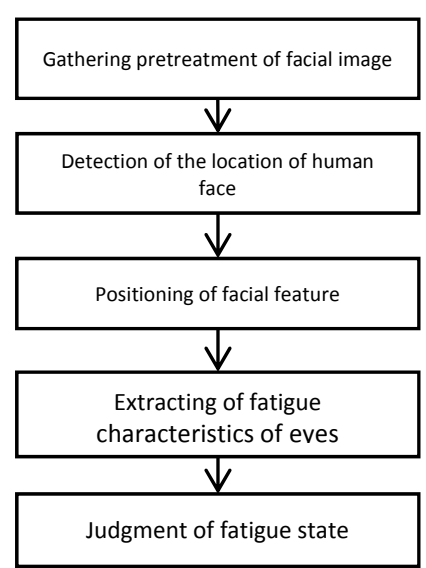

FIGURE I. FATIGUE DRIVING TEST FLOW CHART

\section{FACE DETECTION AND HUMAN FACE FEATURE POINT LOCALIZATION}

A quick positioning of face in an image is the basis of the next work in this paper. Random forest regression model requires a rough position of human face as the initial condition to confirm the existence of face. AdaBoost is a classifier algorithm. The basic idea is to stack a large number of s1imple classifier with general classification ability and form a strong classifier with strong classification ability. When the Adaboost is applied to the face detection, tremendous one-dimensional feature are extracted from the face to form the weak classifiers and then these weak classifier are combined into the final strong classifier. Facial feature points are the critical point that most reflect individual difference in face, including eyebrows contour point, eye contour point, pupil, nasal tip, nostril, mouth contour point and human face contour points, etc. These points are combined to represent the shape of human face. The 68-point of face shape in this paper is shown in Figure II. 


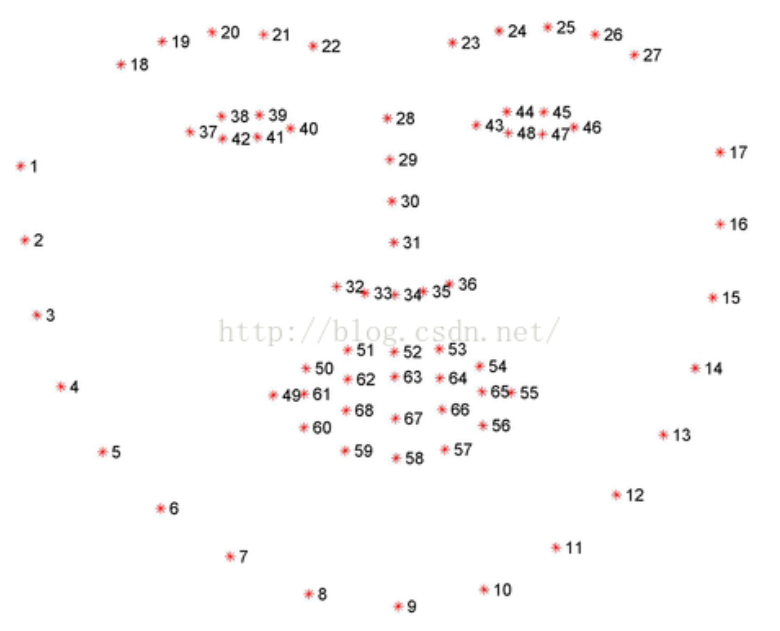

FIGURE II. 68 POINTS FACE SHAPE

The face shape $\mathrm{S}$ is composed of $\mathrm{n}$ feature point, which can be expressed as $S=\left[x_{1}, y_{1}, \ldots, x_{N}, y_{N}\right]^{T}$, this paper $\mathrm{n}=68$. Given the face image, facial feature point localization is to estimate the face shape $\mathrm{S}$, so as to close the real face shape $\hat{S}_{\text {and }}$ minimize the formula (1).

$$
\|S-\hat{S}\|_{2}
$$

The method for estimating $\mathrm{S}$ are mainly optimization methods such as ASM[2] and regression method such as ESR[3] The optimization method represented by ASM uses a large number of examples to train the shape statistic model of face and then searches the matching face feature points according to the texture information around the feature points and complete the facial feature point localization. The regression model based on stochastic forest model is used in this paper.

\section{FATIGUE FEATURE EXTRACTION AND FATIGUE STATE JUDGMENT}

The main flow of driver fatigue detection program is shown in Figure 3. The acquired face image is preprocessed first, then the face detection and face feature point are positioned according to the previous chapter. Because it is difficult to judge the fatigue state by single face image, this paper makes a comprehensive judgment of driver's fatigue state by the change of eye parameters in a period time. In this paper, it takes about 30ms to process each frame image of realized fatigue detection program and the fatigue state of driver is judged by the change of eye parameters within 300 frames. If the driver is found to be fatigue, the system will warn the driver about 10 s and it has high practicality.

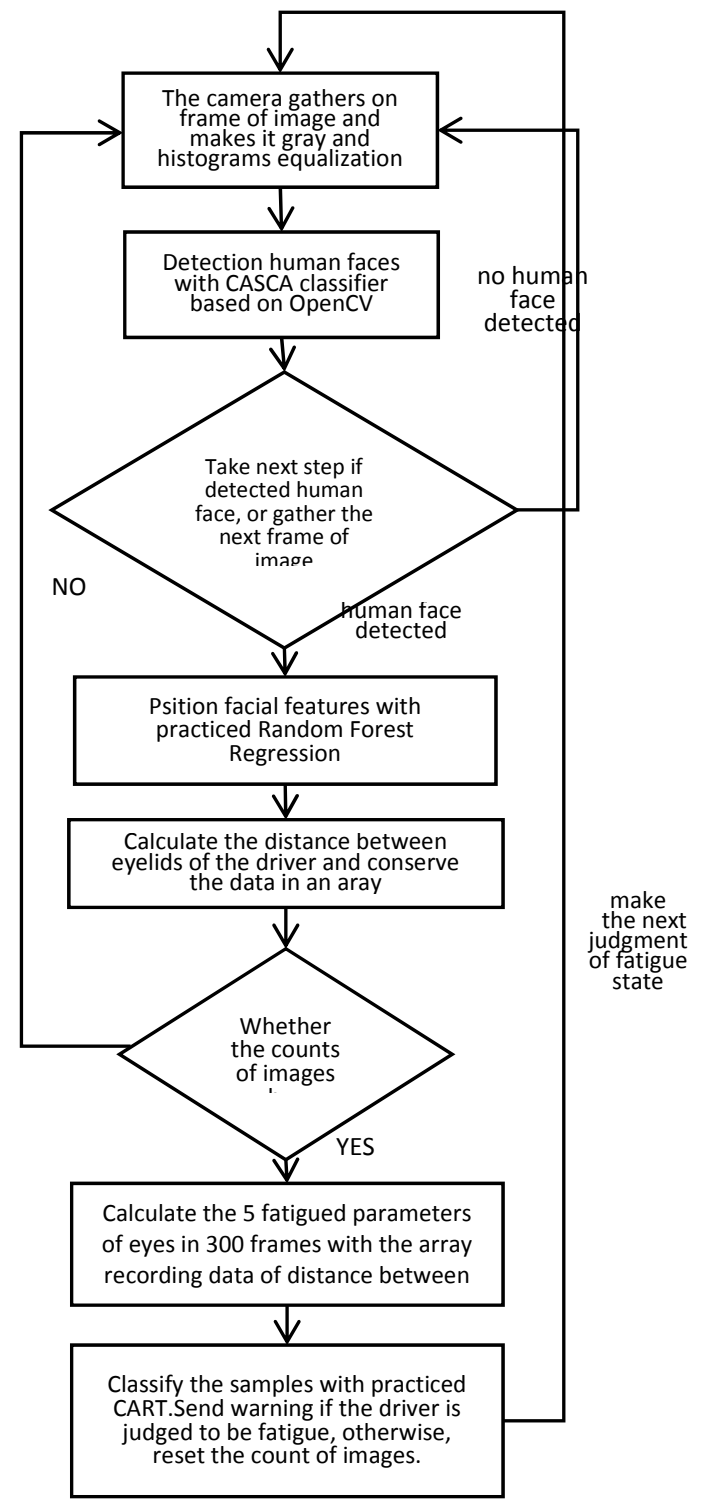

FIGURE III. THE FLOW CHART OF FATIGUE DETECT PROGRAM

\section{A. State Parameters and Their Initialization}

The most direct reaction when people are in the state of fatigue is on the eye. The position of eye can be precisely determined after marking the characteristic point by random forest regression model. After the eyes were positioned, the eye characteristics were extracted for a certain period of time to judge the fatigue state of the driver.

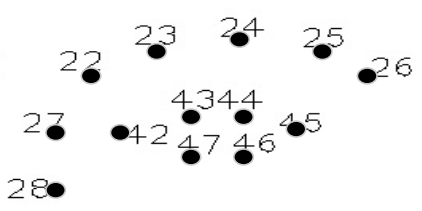

FIGURE IV. THE DISTRIBUTION OF FEATURE POINTS AROUND THE HUMAN EYE (RIGHT) 
In order to avoid the influence of individual factors on the fatigue characteristic, the robust fatigue characteristic need to be extracted. The ocular fatigue characteristics extracted in this paper are the opening and closing degree of the human eyes (the distance between upper and lower eyelid), the distance from 43rd feature point (A point) to 47th feature point (B point) or 44th feature point to 46th feature point. Due to different drivers' eye differences, the distance between eyelids in normal condition varied from person to person. So as to this paper first calculates the average distance between $\mathrm{A}$ and $\mathrm{B}$ point under the current test of 60 frames as the normal opening degree of the current test person's eyes. In this paper, the frame rate of eye positioning is about 30fps. Therefore, taking 10s (300 frames) of image as a time window, in this time window the driver's fatigue stated was judged. If the distance between A and B point is less than $20 \%$ of normal value, we consider that the eye is closed. If the distance between $\mathrm{A}$ and $\mathrm{B}$ point is more than $60 \%$ of the normal value, we consider that the eye is half open and half closed. The fatigue characteristic parameters of human eye are calculated by the statistic of the eye state in the time window and then the fatigue state of driver is judged.

\section{B. Fatigue Feature Parameters}

At present, the method of judging the driver's fatigue state by using eye fatigue characteristic is judged by PERCLOS values[4]. In this paper, the number of frames in the closed state is $x$, the number of frame in the Half-open state is $y$, and the PERCLOS value $\mathrm{P}$ can be calculated by formula (2).

$$
\mathrm{p}=\frac{x}{x+y}
$$

In order to improve the accuracy of driver fatigue identification, the four parameters related to the human eyelid movement were added on the basis of calculating PERCLOS value, which were Maximum Closing Duration (MCD), Blink Frequency (BF), Average Opening Level (AOL) and Time Window Length Corresponding to a Certain value of Closure (TWLCLOS). Decision tree was used to judge the fatigue state of driver by calculating these five parameters in a time window. The decision tree need to be constructed by fatigue samples. This paper uses the expert scoring method based on the facial video to evaluate the fatigue state of driver off-line which means to obtain samples of the decision tree of fatigue judgment. This paper divided the driver's face video into a 10 s video segment, each video about 300 frames, and divided these video into two types of sober and fatigue by the hand-labeled methods By establishing numerous samples, using the decision tree classification of OpenCV to build decision tree of driver's fatigue state, the decision tree is used to judge the fatigue state of driver in the new video clips.

\section{The Classification Regression Tree Training}

In this paper, the CART algorithm (classification and regression tree) is used to discriminate the fatigue status of drivers. A large quantity of fatigue features samples are needed to practice the CART classifier. 120 driving simulations videos of 10 people were gathered through driving simulations experiments and the corresponding average distance of the binocular eyelid was saved as a .csv file as a frame number for the timeline record. And then, the state of drivers were evaluated by watching the videos. The corresponding labels of csv documents were set 1 when drivers are sober, otherwise, they were set 2 . After getting the eyelid distance data with the frame number as the time axis, we can calculate the corresponding five parameters of PERCLOS, MCD, BF, AOL and TWLCLOS. The sample data consisting of these five parameters is also saved to the. Csv file and labeled with the corresponding fatigue status label. The 120 sample data was divided into two parameters of which $70 \%$ as practicing data and $30 \%$ ad testing data. Finally, the CART decision tree classifier based on OpenCV was used to practice the practicing data.

\section{EXPERIMENT AND ANALYSIS}

The experimental platform is an Intel(R) Core(TM) i7$6700 \mathrm{~K}$, Ubuntu operating system with a $640 * 480$ resolution ratio USB camera. The fatigue sample acquisition program, CART decision tree training program and fatigue state judgment program are all based on OpenCV3.1 in C++ implementation.

In this paper, we first use the Fatigue sample acquisition program to collect the 120 sample data of the CART decision tree Training and label it. Then we call the CART Decision tree training program to train the sample data to generate the decision tree of fatigue judgment. At present, when most papers judge the fatigue characteristics after extracting the facial feature points, the PERCLOS value is mainly used to judge whether drivers are fatigued. Besides using this method, we compared these two methods on the basis of the data set. The results of experiment are shown in Table I.

TABle I. COMPARISON OF THE EXPERIMENTAl RESUlts of THE CART DECISION TREe METHOD With THE PERCLOS METHOD

\begin{tabular}{|c|c|c|c|c|}
\hline Different algorithm & \multicolumn{2}{|c|}{ The method of this paper } & \multicolumn{2}{|c|}{ PERCLOS } \\
\hline Real state & sober & fatigue & sober & fatigue \\
\hline sober & 56 & 4 & 51 & 9 \\
\hline fatigue & 2 & 58 & 11 & 49 \\
\hline False alarm rate in sober & \multicolumn{2}{|c|}{6.7} & \multicolumn{2}{|c|}{15.0} \\
\hline False alarm rate in fatigue & \multicolumn{2}{|c|}{3.3} & \multicolumn{2}{|c|}{18.3} \\
\hline Accuracy rate (\%) & \multicolumn{2}{|c|}{95.0} & \multicolumn{2}{|c|}{83.3} \\
\hline
\end{tabular}


Compared with PERCLOS method, the method of decision trees synthesizing several characteristic parameters of eyes in the paper has higher accuracy rate and lower false alarm rate, which can be seen in table1. When the is in the state of fatigue, the false alarm rate of method in the paper is low to $3.3 \%$,and the early warning will be sent to drivers in 10 seconds. They meet the performance requirements of fatigue detection.

\section{CONCLUSION}

In the paper, we combined random forest regression model and CART decision tree. Random forest regression model was used to obtain 68 features of human face. And then five parameters of human eyes in videos with 300 frames including PERCLOS, PERCLOS, MCD, BF, AOL and TWLCLOS were extracted .After that, the judgment of whether the drivers in videos have state of fatigue were made with CART decision tree. In the results, the accuracy of the driver fatigue detection algorithm proposed in the paper is $95 \%$, and the error rate is $3.3 \%$ when the fatigue occurs, which satisfies the practical use.

\section{ACKNOWLEDGEMENT}

I am grateful to all the people who have participated in the work of this paper. This work is supported by the National High Technology Research and Development Program [grant numbers 2013AA12A206].

\section{REFERENCES}

[1] Viola P, Jones M J. Robust Real-Time Face Detection[M]. Kluwer Academic Publishers, 2004.

[2] Li Y, Da F, Automation S O, et al. Expression-insensitive 3D face recognition method based on facial fiducial points[J]. Journal of Image \& Graphics, 2014.

[3] Jiang J G, Wang L, Mei-Bin Q I, et al. Detection of fatigue driving based on eye state[J]. Journal of Hefei University of Technology, 2015.

[4] ZHAO Xiaolin,WANG Shigang, et al. Driver Fatigue Detection Based on Eye State[J]. 2015, 33(4): 449-453. 